\title{
A logistic model to predict early pregnancy loss following in vitro fertilization based on 2601 infertility patients
}

\author{
Yan Yi ${ }^{1}$, Guangxiu Lu, ${ }^{1,2}$, Yan Ouyang ${ }^{1,2}$, Ge lin ${ }^{1,2}$, Fei Gong ${ }^{1,2}$ and Xihong Li ${ }^{2 *}$
}

\begin{abstract}
Background: According to previous studies, even after embryonic cardiac activity is detected, the pregnancy loss rate remains 3-4\%. The objectives of this study were to investigate the differences in ultrasound parameters between a miscarriage group and an ongoing pregnancy group during the $1^{\text {st }}$ trimester and to build a logistic model to predict early pregnancy loss (EPL) after the appearance of embryonic cardiac activity in patients who have undergone in vitro fertilization embryo transfer (IVF-ET) treatment.
\end{abstract}

Method: A total of 2601 patients with early singleton pregnancies with embryonic cardiac activity were retrospectively analyzed after IVF from January 2010 to June 2011. Transvaginal sonography (TVS) was performed at 6 to 10 weeks of gestational age (GA). The mean gestational sac diameter (MSD), crown-rump length (CRL), fetal heart rate (FHR), and yolk sac diameter (YSD) were measured by TVS.

Results: A total of 2400 patients had an ongoing pregnancy and an additional 201 (7.7\%) patients miscarried during the first trimester after fetal cardiac activity had been established. The maternal age (MA) and infertility duration were much greater, and the MSD, CRL, and FHR were much lower in the miscarriage group than in the ongoing pregnancy group after IVF $(P<0.05)$. The prediction model utilized the following equation: the possibility of $E P L=\exp (z)$ / $(1+\exp (z))$, where $z=-21.456+(0.114 \times M A)+(4.305 \times \times G A)-(0.043 \times M S D)-(0.359 \times C R L)-(0.091 \times F H R)+2.243$ (fluid collection present around the gestational sac (GS)) +2.519 (when YSD < 3) or -0.347 (when YSD > 5.5).

Conclusion: The MA, MSD, CRL, YSD, FHR, infertility duration, and fluid collection around the GS were each correlated with EPL after IVF in infertile patients. A logistic model is a useful tool for predicting EPL after the appearance of embryonic cardiac activity (area under the curve $[\mathrm{AUC}]=0.909$ ).

Keywords: Logistic model, Early pregnancy loss, Mean gestational sac diameter, Crown-rump length, Fetal heart rate, In vitro fertilization embryo transfer

\section{Background}

With the development of transvaginal sonography (TVS), more detailed information may be obtained during early pregnancy [1]. Specifically, the fetal heart rate (FHR) may be measured precisely using M mode TVS [2]. According to previous studies, the spontaneous $1^{\text {st }}$ trimester miscarriage rate is $15-30 \%$ in the general population [3, 4]. Even after embryonic cardiac activity is detected, the pregnancy loss rate remains 3-4\% [5]. Therefore, the development of a prediction model for early pregnancy loss (EPL) is

\footnotetext{
* Correspondence: xihongliyxk@163.com

${ }^{2}$ Reproductive and Genetic Hospital of CITIC-Xiangya, Xiangya Road,

Changsha 410078, Hunan, P. R. China

Full list of author information is available at the end of the article
}

necessary after the appearance of fetal cardiac activity, particularly in infertility patients, who experience tremendous psychological stress and anxiety.

Many parameters can be used to provide a prognosis of pregnancy outcome, and these types of parameters, such as maternal age (MA) and sonographic measurements, are also easily attainable. Fetal bradycardia has been correlated with EPL [6]. One study reported that an FHR less than 90 beats per minute (bpm) is associated with poor pregnancy outcomes [5]. The measurement of the yolk sac size using TVS is also valuable for predicting EPL. An excessively large or small yolk sac, as well as the absence of a yolk sac, is associated with 
adverse pregnancy outcomes [7, 8]. The ongoing pregnancy rate was much greater in the group in which the yolk sac diameter (YSD) ranged from 2 to $6 \mathrm{~mm}$ than that in groups in which the YSD was either less than $2 \mathrm{~mm}$ or greater than $6 \mathrm{~mm}(\mathrm{OR}=33.1, P<0.001)$ [9]. The mean gestational sac diameter (MSD) and crownrump length (CRL) are also predictors of EPL [10].

A prediction model including the above four parameters has been established for females who undergo spontaneous conception $[2,11]$. When these indicators are used to predict EPL, the gestational age (GA) should also be taken into consideration because the MSD, CRL, YSD, and FHR vary depending on GA. However, for spontaneous conception, the exact GA cannot be determined in females whose last menstrual period was uncertain or whose menstrual cycle is irregular. Thus, in a previous study, the GA was not included in the prediction model, which led to the reduced prediction power of this model. Therefore, the aim of our study was to establish a precise prediction model of EPL in females after IVF-ET treatment because an accurate GA can be determined in this population, increasing the prediction power of the model.

In addition, no large studies have been conducted to verify the relationships between early ultrasound parameters and EPL in infertility patients after IVF. Therefore, our study analyzed the relationship between ultrasound factors and EPL based on data collected from initial TVS scans of 2601 females with viable singleton pregnancies who underwent IVF-ET in our hospital. Using these data, we designed a valuable model to predict EPL, even if embryonic cardiac activity was detected initially.

\section{Methods}

The institutional review board approved this study before data collection. A retrospective chart review was conducted for 4476 infertile patients to identify 2601 patients who had positive fetal cardiac acitivty on their first ultrasound for inclusion into our analysis. All these patients underwent IVF-ET treatment at the Reproductive and Genetic Hospital of CITIC-Xiangya from January 2010 to June 2011.

These 4476 patients underwent controlled ovarian hyperstimulation (using follicle-stimulating hormone or human menopausal gonadotropin), human chorionic gonadotropin (HCG) injection, and blood tests for serum $\beta$-HCG 14 days after embryo transfer (ET). A total of 2806 patients had positive results for serum $\beta$-HCG. TVS was subsequently performed on these patients to measure the MSD, CRL, YSD, and FHR and to observe fluid collection around the GS from 6 to 10 weeks of GA. The GS was measured in three orthogonal planes, and the data were recorded as the mean gestational diameter. The yolk sac was measured along its longest diameter using a caliper placed along the center wall of the yolk sac [12]. The CRL was measured along the greatest length of the embryo. The FHR was measured using $M$ mode TVS. The ultrasound devices used in this study consisted of either a GE VOLUSON 730 or an E8 (General Electric Tech Co., Ltd., New York, America) instrument housed in the imaging department of our hospital, and all examinations were performed by trained sonographers. A total of 2601 patients exhibited viable (fetal heart activity detected) singleton pregnancies in the initial TVS scan conducted during this study. Multiples were excluded from this study. The MA, infertility duration, sonographic data and early pregnancy outcome of these patients were collected in this study. EPL was defined as miscarriage before or at 12 weeks of gestation and ongoing pregnancy was defined as pregnancy > 12 weeks of gestation.

The differences in the clinical characteristics between the miscarriage group and the ongoing pregnancy group were compared using either a two-sample $t$-test or a Wilcoxon rank sum test. The fertility duration and IVF cycle are expressed as medians [interquartile range] and were compared using the Wilcoxon rank sum test. The MSD, CRL, FHR and YSD in the EPL group and the ongoing pregnancy group were compared during each gestational week using two-sample t-tests and chi-square tests. A logistic regression model was established to assess the odds of pregnancy loss using the ultrasound parameters and additional measurements. A receiver operating characteristic (ROC) curve was obtained to assess the predictive value of this model and the individual ultrasound indicators. The significance level (alpha) was $P<0.05$ for all analyses. Statistical Package for Social Sciences version 18 (SPSS Inc., Chicago, IL, USA) was used for the data analysis.

\section{Results}

A total of 2400 (92.27 \%) patients had an ongoing pregnancy and an additional 201 (7.7\%) patients miscarried during the first trimester after fetal cardiac activity appearance. Both the mean MA $(32.67 \pm 4.35$ vs. $30.59 \pm 4.27, P<0.001)$ and infertility duration $(4[3-7]$ vs. $5[3-8.5], P=0.03)$ were significantly greater in the miscarriage group than in the ongoing pregnancy group. However, the number of IVF treatment cycles was not different between the two groups, and most of the patients in this study underwent only one IVF transfer cycle $(1[1,2]$ vs. $1[1,2], P=0.93)$.

The MSD, CRL and FHR were compared between the EPL group and the ongoing pregnancy group during each gestational week (Table 1). In the ongoing pregnancy group, the MSD, CRL and FHR were significantly higher than the corresponding values in the EPL group from 6 to 9 weeks of gestation (Table $1, P<0.05$ ). In addition, the FHR increased with GA from 6 to 9 weeks and peaked at approximately 9 weeks (Fig. 1). 
Table 1 Comparison of the MSD, CRL and FHR in the two groups during each gestational week

\begin{tabular}{|c|c|c|c|c|}
\hline \multirow[t]{2}{*}{ Parameters } & \multirow{2}{*}{$\begin{array}{l}\text { Ongoing pregnancy } \\
n=2400\end{array}$} & Early pregnancy loss & \multirow[t]{2}{*}{$P$} & \multirow[t]{2}{*}{$95 \% \mathrm{Cl}$} \\
\hline & & $n=201$ & & \\
\hline \multicolumn{5}{|l|}{ MSD (mm) } \\
\hline 6 week & $19.95 \pm 3.64$ & $17.50 \pm 4.82$ & $<0.01$ & $(1.42,3.47)$ \\
\hline 7 week & $26.42 \pm 5.48$ & $19.06 \pm 6.68$ & $<0.01$ & $(5.76,8.97)$ \\
\hline 8 week & $35.70 \pm 5.89$ & $24.39 \pm 8.2$ & $<0.01$ & $(7.61,15.02)$ \\
\hline 9 week & $41.16 \pm 6.16$ & $30.00 \pm 8.94$ & 0.006 & $(4.26,18.07)$ \\
\hline 10 week & $48.59 \pm 6.27$ & - & - & - \\
\hline \multicolumn{5}{|l|}{ CRL (mm) } \\
\hline 6 week & $3.93 \pm 1.134$ & $3.30 \pm 1.05$ & $<0.01$ & $(0.383,0.863)$ \\
\hline 7 week & $9.56 \pm 2.34$ & $5.22 \pm 2.77$ & $<0.01$ & $(3.672,5.011)$ \\
\hline 8 week & $18.57 \pm 2.58$ & $8.17 \pm 5.3$ & $<0.01$ & $(8.030,12.774)$ \\
\hline 9 week & $24.50 \pm 2.57$ & $13.93 \pm 11.6$ & 0.026 & $(1.645,19.489)$ \\
\hline 10 week & $34.98 \pm 3.35$ & - & - & - \\
\hline \multicolumn{5}{|l|}{ FHR (bpm) } \\
\hline 6 week & $115.64 \pm 9.25$ & $98.12 \pm 17.66$ & $<0.01$ & $(15.421,19.606)$ \\
\hline 7 week & $144.31 \pm 12.36$ & $97.26 \pm 32.68$ & $<0.01$ & $(39.665,54.427)$ \\
\hline 8 week & $172.85 \pm 9.12$ & $100.86 \pm 40.74$ & $<0.01$ & $(53.887,90.078)$ \\
\hline 9 week & $175.89 \pm 9.27$ & $118.44 \pm 50.91$ & 0.010 & $(18.305,96.594)$ \\
\hline 10 week & $172.31 \pm 9.21$ & - & - & - \\
\hline
\end{tabular}

Comparisons were made using a two sample $t$-test

Values are expressed as the means \pm standard deviation

$\mathrm{Cl}$ confidence interval

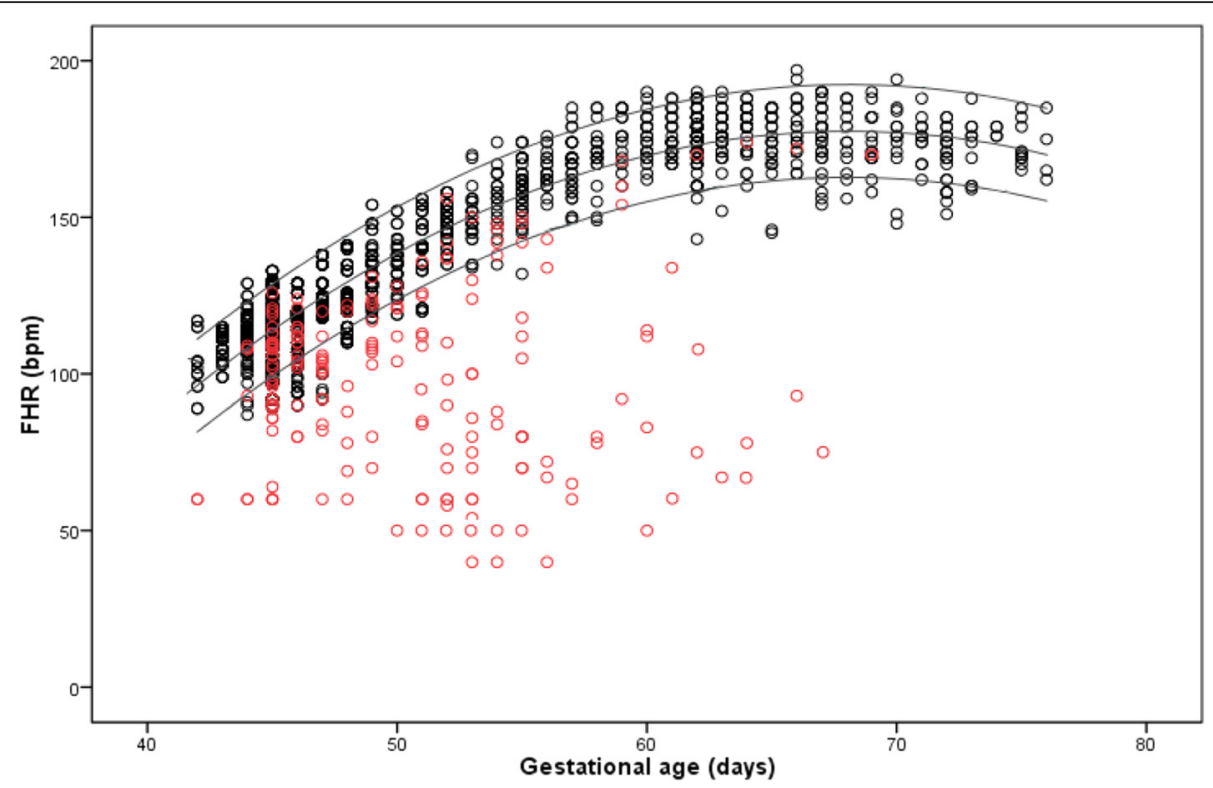

Fig. 1 Scatterplot of the changes in fetal heart rate (FHR) for ongoing pregnancy (black circles) and early pregnancy loss (red circles) from 6 to 10 weeks of gestation. The normal range (black line) was derived from the ongoing pregnancy group (median, $95^{\text {th }}$ and $5^{\text {th }}$ percentile) 
Table 2 Comparison of the YSD and fluid collection around the GS between the two groups

\begin{tabular}{cclll}
\hline Parameters & Total & $\begin{array}{l}\text { Ongoing pregnancy } \\
\mathrm{n}=2400\end{array}$ & $\begin{array}{l}\text { Early pregnancy loss } \\
\mathrm{n}=201\end{array}$ & $\mathrm{P}$ \\
\hline $\begin{array}{ccccc}\text { YSD }(\mathrm{mm}) \\
<3\end{array}$ & 19 & $9(47.4 \%)$ & $10(52.6 \%)$ & \\
$3-5.5$ & 2420 & $2251(93.0 \%)$ & $169(7.0 \%)$ & $<0.001$ \\
$>5.5$ & 162 & $140(86.4 \%)$ & $22(13.6 \%)$ & \\
Fluid collection around GS & & $<0.001$ \\
No & 2188 & $2077(94.9 \%)$ & $111(5.1 \%)$ & \\
Yes & 413 & $323(78.2 \%)$ & $90(21.8 \%)$ & \\
\hline
\end{tabular}

Comparisons were made using the chi-square test

Significant differences were observed in the ongoing pregnancy rate among different YSD ranges (Table 2). The ongoing pregnancy rate was higher $(93 \%)$ in the $3-$ $5.5 \mathrm{~mm}$ YSD group than in any other YSD group. In the $\mathrm{YSD}<3 \mathrm{~mm}$ group, the ongoing pregnancy rate was $47.4 \%$. The miscarriage rate was $21.8 \%$ in patients exhibiting fluid collection around the GS, which was significantly higher than in patients without fluid collection $(P<0.001)$.

A logistic regression model was established to assess the effect of the MA, GA, MSD, CRL, FHR, YSD, and fluid collection around the GS (Table 3). Infertility duration was excluded from this model due to stepwise screening. According to this logistic model, the probability of first trimester miscarriage was equal to the following: $\exp (\mathrm{z}) /(1+\exp (\mathrm{z}))$, where $\mathrm{z}=-21.456+(0.114 \times \mathrm{MA})+(4.305 \times \mathrm{GA})-(0.043 \times$ MSD) - $(0.359 \times$ CRL $)-(0.091 \times$ FHR $)+2.243$ (fluid collection present around the GS) +2.519 (when YSD $<3$ ) or -0.347 (when YSD $>5.5$ ).
ROC curve analysis was used to evaluate the predictive value of this logistic model and several individual indicators (MA, MSD, CRL and FHR). Figure 2 depicts the area under the curve (AUC) of the logistic model $(\mathrm{AUC}=0.909)$ and that for the FHR $(\mathrm{AUC}=0.800)$, MSD $\quad(\mathrm{AUC}=0.675), \quad \mathrm{MA} \quad(\mathrm{AUC}=0.634)$ and $\mathrm{CRL}$ $(\mathrm{AUC}=0.623)$.

\section{Discussion}

Regarding the diagnostic criteria for nonviable pregnancy during the $1^{\text {st }}$ trimester, a previous study used the following guideline for EPL: an MSD $\geq 25 \mathrm{~mm}$ and either no embryo or a CRL $\geq 7 \mathrm{~mm}$ and no heartbeat [10]. This guideline applies to early pregnancy without fetal cardiac activity. However, for some cases, even after cardiac activity appears initially, there is still a chance that the embryo will be lost. Most of these patients had unfavorable ultrasound parameters such as small GS and CRL. Therefore, it is necessary for clinicians to identify cases that carry a risk of pregnancy failure because repeated TVS is recommended, especially for infertility patients after IVF-ET, in these cases. Therefore, our study established an accurate model to predict EPL after the detection of cardiac activity in infertility patients after IVF. This model includes ultrasound parameters, such as the MSD, CRL, YSD and FHR, which correspond to an accurate GA. According to the ROC curve analysis, the AUC of the logistic model (AUC $=0.909$ ) was greater than that of any other individual parameter.

In this study, a lower FHR was associated with EPL after the use of reproductive assistance technology. A lower FHR may result from an imbalance in the autonomic nervous system [13] and may be correlated with chromosomal anomalies [14]. In trisomy 18, the FHR is

Table $\mathbf{3}$ Logistic regression analysis of the indicators used to predict EPL

\begin{tabular}{llll}
\hline Parameters & $\beta$ & OR (Cl) & $P$ \\
\hline MA (years) & 0.114 & $1.121(1.066-1.178)$ & $74.088(32.957-166.551)$ \\
GA (weeks) & 4.305 & $0.958(0.910-1.008)$ \\
MSD (mm) & -0.043 & $0.699(0.619-0.789)$ \\
CRL (mm) & -0.359 & $0.913(0.898-0.929)$ \\
FHR (bpm) & -0.091 & & $<0.001$ \\
YSD (mm) & & 1 & $12.421(3.127-49.343)$ \\
$3-5.5$ & & $0.707(0.255-1.965)$ & $<0.001$ \\
$<3$ & 2.519 & $9.422(6.099-14.558)$ & $<0.001$ \\
$>5.5$ & -0.347 & & $<0.001$ \\
Fluid collection around the GS & 2.243 &
\end{tabular}

The data were analyzed using a binary logistic regression analysis

Method $=$ stepwise (LR). Criteria $=$ PIN (0.05) and POUT (0.10). Model $R^{2}=0.593, P<0.001$

Dependent variable assignment: ongoing pregnancy $=0$, miscarriage $=1$

Independent variable assignment: YSD $\{\mathrm{mm}, 3-5.5=1$ (Dummy variable), $<3=2,>5.5=3\}$; fluid collection around GS (yes $=1$, no $=0$ )

$O R$ odds ratio, $\mathrm{Cl}$ confidence interval 


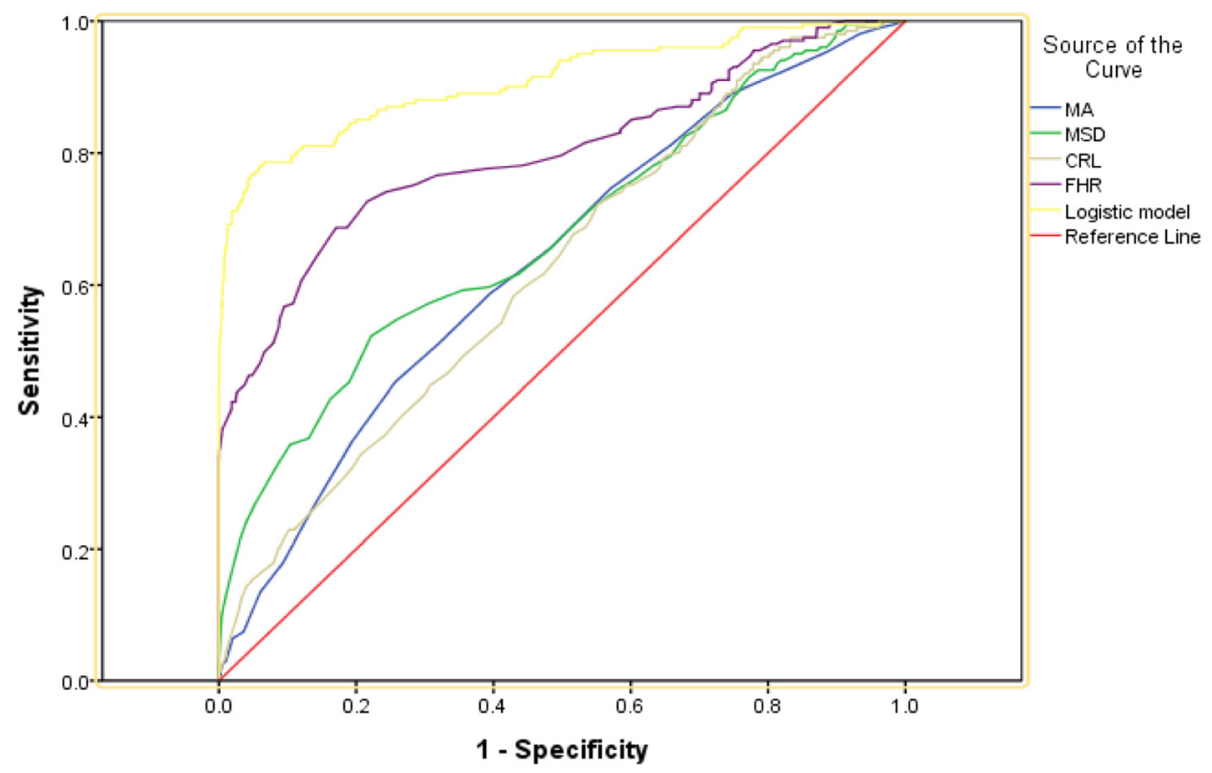

Fig. 2 ROC curve analysis for the MA, MSD, CRL, FHR and logistic model for EPL prediction

decreased; a lower FHR may indicate a pre-terminal event due to severe early-onset growth restriction [15]. Based on the change in FHR in conjunction with the GA in the ongoing pregnancy group (Fig. 1), the FHR peaked at approximately $175.89 \mathrm{bpm}$ at 9 weeks of gestation. However, it is unknown whether parameter is abnormal when the FHR is above the normal reference range at 69 weeks of gestation or when the FHR increases with GA even after 9 weeks. The FHR is known to increase in some cases of trisomy 13 at 11-13 weeks of gestation [15]. Trisomy 13 causes left ventricular outflow tract obstruction (LVOTO) [16]; tachycardia may occur as a compensatory mechanism to increase cardiac output [17]. Additional studies focusing on whether fetal tachycardia during the $1^{\text {st }}$ trimester is associated with EPL should be performed.

The yolk sac appearance in the gestational sac is further evidence of an intrauterine pregnancy. Some studies have found no correlation between EPL and either the size or the shape of the yolk sac $[18,19]$. These results are inconsistent with the results of our study. The reasons for this inconsistency may include measurement error or a difference in sample size. The yolk sac normally ranges from 2 to $6 \mathrm{~mm}$, with a narrow distribution; therefore, this parameter is difficult to precisely measure. Furthermore, the measurement methods used in different studies were not the same. One study indicated that the yolk sac was measured in three orthogonal planes, from the outer borders of the sac [20]. In another study, the yolk sac was measured from the inner limits of the longest diameter of the yolk sac on a magnified scan [21]. According to our study, the ongoing pregnancy rate was lower in both the larger and smaller YSD groups than in the normal group. The yolk sac plays an important role in the primary exchange between the mother and embryo before the placental circulation is established. In addition to metabolic substance exchange, the yolk sac also has endocrine and hematopoietic functions during early pregnancy $[22,23]$. A large yolk sac may be due to yolk sac membrane dysfunction, resulting in collection of secretions. A small yolk sac or no yolk sac may be associated with either dysplasia or early atrophy.

Many studies have reported that advanced MA is associated with poor pregnancy outcomes after IVF [24, 25]. Our study is consistent with this view in that the miscarriage rate increased with age. This result may be attributed to the deterioration of the oocyte and to endocrine variations that occur with aging. The mean MAs in the two groups were significantly different; therefore, this variable was selected for inclusion in the logistic regression analysis.

Fluid collection around the gestational sac may indicate either choriodecidual or amniodecidual separation during early pregnancy, with or without vaginal bleeding, and this is sometimes associated with abdominal pain. This fluid collection presents as an anechoic area around the gestational sac and may be detected easily by TVS. According to our logistic model, fluid collection around the GS is negatively correlated with ongoing pregnancy and is a useful prognostic indicator. Perhaps choriodecidual and amniodecidual separation are indirect signs of the maternal immune system attacking the embryo. Further investigation is needed to determine whether the 
size, volume, and position of the fluid collection are related to EPL.

The duration of infertility was excluded from this model partly because it is influenced by several confounding factors and partly because it is also a reflection of MA. Therefore, MA is more of a direct factor than the duration of infertility. In addition, number of IVF treatment cycles may not completely reflect the ovarian response because low and high ovarian responses, as in ovarian hyper-stimulation syndrome (OHSS), may both result in transfer cycle cancellation. Therefore, the number of treatment cycles was not included in this model.

One deficiency of this study was that fewer samples were included in the miscarriage group than in the ongoing pregnancy group. Another limitation of this study was that only singleton pregnancies were included. If the multiples were also included in this study, the result analysis will be complicated. First, we need to consider how to record and analyze monochorionic twin or triplet pregnancy (one gestational sac with multiple embryo and yolk sac). Besides, measurements error of ultrasound parameters (MSD, CRL, FHR etc.) will increase in multiple pregnancies sometimes. For example, in a dichorionic twins pregnacy, one of the gestational sac implanted in unfavorable observation position just behind another gestational sac which may lead to incorrect mesurements for ultrasound parameters. Besides, not including whether or not the embryo was biopsied for preimplantation genetic screening (PGS) would also be a limitation for this study. Before applying this model in clinical practice, additional prospective investigations should be performed.

\section{Conclusion}

This study provides a logistic model to predict EPL after the appearance of embryonic cardiac activity in infertility patients. When the predictive results suggest risk of EPL, repeated scans are recommended for these patients, which will help clinicians to determine subsequent interventions.

\section{Abbreviations \\ AUC: area under the curve; Bpm: beats per minute; CRL: crown-rump length; EPL: early pregnancy loss; FHR: fetal heart rate; GA: gestational age; GS: gestational sac; IVF-ET: in vitro fertilization embryo transfer; MA: maternal age; MSD: mean gestational sac diameter; OHSS: ovarian hyper-stimulation syndrome; PGS: preimplantation genetic screening; ROC curve: receiver operating characteristic curve; TVS: transvaginal sonography; YSD: yolk sac diameter.}

\section{Competing interests}

The authors declare that they have no competing interests.

\section{Authors' contributions}

$Y Y$ and $X L$ collected the relevant clinical data. $Y Y$ drafted the manuscript. $Y O$ participated in the study design and helped to collect the relevant clinical data. FG and $G L$ helped to collect the relevant clinical data and were involved in revising the manuscript. GL and $Y Y$ conceived the study, participated in its design and coordinated and helped to draft the manuscript. All authors have read and approved the final manuscript.

\section{Author details}

${ }^{1}$ Institute of Reproductive and Stem Cell Engineering, Central South University, Xiangya Road, Changsha 410078, Hunan, P. R. China. ${ }^{2}$ Reproductive and Genetic Hospital of CITIC-Xiangya, Xiangya Road, Changsha 410078, Hunan, P. R. China.

Received: 21 December 2015 Accepted: 4 March 2016

Published online: 31 March 2016

\section{References}

1. Macklon NS, Geraedts JP, Fauser BC. Conception to ongoing pregnancy: the 'black box' of early pregnancy loss. Hum Reprod Update. 2002;8(4):333-43.

2. Papaioannou Gl, Syngelaki A, Maiz N, Ross JA, Nicolaides KH. Ultrasonographic prediction of early miscarriage. Hum Reprod. 2011;26(7): 1685-92.

3. Goldstein SR. Embryonic death in early pregnancy: a new look at the first trimester. Obstet Gynecol. 1994;84(2):294-7.

4. Edmonds DK, Lindsay KS, Miller JF, Williamson E, Wood PJ. Early embryonic mortality in women. Fertil Steril. 1982;38(4):447-53.

5. Rauch ER, Schattman GL, Christos PJ, Chicketano T, Rosenwaks Z. Embryonic heart rate as a predictor of first-trimester pregnancy loss in infertility patients after in vitro fertilization. Fertil Steril. 2009;91(6):2451-4.

6. Doubilet PM, Benson CB, Chow JS. Outcome of pregnancies with rapid embryonic heart rates in the early first trimester. AJR Am J Roentgenol. 2000;175(1):67-9.

7. Jeve $Y$, Rana R, Bhide A, Thangaratinam S. Accuracy of first-trimester ultrasound in the diagnosis of early embryonic demise: a systematic review. Ultrasound Obstet Gynecol. 2011;38(5):489-96.

8. Jauniaux E, Johns J, Burton GJ. The role of ultrasound imaging in diagnosing and investigating early pregnancy failure. Ultrasound Obstet Gynecol. 2005;25(6):613-24.

9. Bae S, Karnitis J. Triple ultrasound markers including fetal cardiac activity are related to miscarriage risk. Fertil Steril. 2011;96(5):1145-8.

10. Doubilet PM, Benson CB, Bourne T, Blaivas M, Barnhart KT, Benacerraf BR, Brown DL, Filly RA, Fox JC, Goldstein SR, et al. Diagnostic criteria for nonviable pregnancy early in the first trimester. N Engl J Med. 2013;369(15):1443-51.

11. Bottomley C, Van Belle V, Kirk E, Van Huffel S, Timmerman D, Bourne T. Accurate prediction of pregnancy viability by means of a simple scoring system. Hum Reprod. 2013;28(1):68-76.

12. Jauniaux $E$, Jurkovic $D$, Henriet $Y$, Rodesch F, Hustin J. Development of the secondary human yolk sac: correlation of sonographic and anatomical features. Hum Reprod. 1991;6(8):1160-6.

13. Hanprasertpong T, Phupong $\mathrm{V}$. First trimester embryonic/fetal heart rate in normal pregnant women. Arch Gynecol Obstet. 2006;274(5):257-60.

14. Montenegro N, Ramos C, Matias A, Barros H. Variation of embryonic/fetal heart rate at 6-13 weeks' gestation. Ultrasound Obstet Gynecol. 1998; 11(4):274-6.

15. Papaioannou GK, Syngelaki A, Maiz N, Ross JA, Nicolaides KH. Sonographic markers of aneuploidies at 6-10 weeks of gestation. Early Hum Dev. 2011; 87(7):453-6.

16. Hyett J, Moscoso G, Nicolaides K. Abnormalities of the heart and great arteries in first trimester chromosomally abnormal fetuses. Am J Med Genet. 1997;69(2):207-16

17. Rudolph AM, Heymann MA. Cardiac output in the fetal lamb: the effects of spontaneous and induced changes of heart rate on right and left ventricular output. Am J Obstet Gynecol. 1976;124(2):183-92.

18. Tan S, Ipek A, Pektas MK, Arifoglu M, Teber MA, Karaoglanoglu M. Irregular yolk sac shape: is it really associated with an increased risk of spontaneous abortion? J Ultrasound Med. 2011;30(1):31-6.

19. Reece EA, Scioscia AL, Pinter E, Hobbins JC, Green J, Mahoney MJ, Naftolin F. Prognostic significance of the human yolk sac assessed by ultrasonography. Am J Obstet Gynecol. 1988;159(5):1191-4.

20. Bottomley C, Bourne T. Dating and growth in the first trimester. Best Pract Res Clin Obstet Gynaecol. 2009;23(4):439-52.

21. Tan S, Pektas MK, Ozcan AS, Akcay Y, Ozat M, Arslan H. Frequency of a persistent yolk sac and its relationship with the gestational outcome. J Ultrasound Med. 2012;31(5):697-702.

22. Pereda TJ, Motta PM. New advances in human embryology: morphofunctional relationship between the embryo and the yolk sac. Med Electron Microsc. 1999;32(2):67-78. 
23. Lindsay DJ, Lovett IS, Lyons EA, Levi CS, Zheng XH, Holt SC, Dashefsky SM. Yolk sac diameter and shape at endovaginal US: predictors of pregnancy outcome in the first trimester. Radiology. 1992;183(1):115-8.

24. Nybo AA, Wohlfahrt J, Christens P, Olsen J, Melbye M. Maternal age and fetal loss: population based register linkage study. BMJ. 2000;320(7251): 1708-12.

25. Tummers $P$, De Sutter $P$, Dhont M. Risk of spontaneous abortion in singleton and twin pregnancies after IVF/ICSI. Hum Reprod. 2003; 18(8):1720-3.

Submit your next manuscript to BioMed Central and we will help you at every step:

- We accept pre-submission inquiries

- Our selector tool helps you to find the most relevant journal

- We provide round the clock customer support

- Convenient online submission

- Thorough peer review

- Inclusion in PubMed and all major indexing services

- Maximum visibility for your research

Submit your manuscript at www.biomedcentral.com/submit 